\title{
Dependence of the L- to H-mode Power Threshold on Toroidal Rotation and the Link to Edge Turbulence Dynamics
}

\author{
G.R. McKee 1), P. Gohil 2), D.J. Schlossberg 1) J.A. Boedo 3) K.H. Burrell 2)
}

J.S. deGrassie 2). R.J. Groebner 2), M.A. Makowski 4), R.A. Moyer 3), C.C. Petty 2),

T.L. Rhodes 5), L. Schmitz 5), M.W. Shafer 1), W.M. Solomon 6), M. Umansky 4),

G. Wang 5), A.E. White 5), and X. Xu 4)

1) University of Wisconsin-Madison, Madison, Wisconsin 53706, USA

2) General Atomics, PO Box 85608, San Diego, California 92186-5608, USA

3) University of California-San Diego, La Jolla, California 92093, USA

4) Lawrence Livermore National Laboratory, Livermore, California, USA

5) University of California-Los Angeles, Los Angeles, California, USA

6) Princeton Plasma Physics Laboratory, Princeton, New Jersey, USA

e-mail contact of main author: mckee@fusion.gat.com

\begin{abstract}
The injected power required to induce a transition from L-mode to H-mode plasmas is found to depend strongly on the injected neutral beam torque and consequent plasma toroidal rotation. Edge turbulence and flows, measured near the outboard midplane of the plasma $(0.85<\mathrm{r} / \mathrm{a}<1.0)$ on DIII-D with the highsensitivity 2D beam emission spectroscopy (BES) system, likewise vary with rotation and suggest a causative connection. The L-H power threshold in plasmas with the ion $\nabla \mathrm{B}$ drift away from the X-point decreases from 4-6 MW with co-current beam injection, to 2-3 MW with near zero net injected torque, and to $<2 \mathrm{MW}$ with counter injection. Plasmas with the ion $\nabla \mathrm{B}$ drift towards the X-point exhibit a qualitatively similar though less pronounced power threshold dependence on rotation. 2D edge turbulence measurements with BES show an increasing poloidal flow shear as the L-H transition is approached in all conditions. At low rotation, the poloidal flow of turbulent eddies near the edge reverses prior to the L-H transition, generating a significant poloidal flow shear that exceeds the measured turbulence decorrelation rate. This increased poloidal turbulence velocity shear may facilitate the L-H transition. No such reversal is observed in high rotation plasmas. The poloidal turbulence velocity spectrum exhibits a transition from a Geodesic Acoustic Mode zonal flow to a higher-power, lower frequency, zero-mean-frequency zonal flow as rotation varies from co-current to balanced during a torque scan at constant injected neutral beam power, perhaps also facilitating the L-H transition. This reduced power threshold at lower toroidal rotation may benefit inherently low-rotation plasmas such as ITER.
\end{abstract}

\section{Introduction}

Magnetically confined tokamak plasmas undergo a transition from a low-confinement mode, L-mode, to a high-confinement mode, H-mode [1] when the power flow through the boundary exceeds a threshold. The power threshold required to trigger this L-H transition has been empirically studied on numerous tokamak experiments and is a well-known quantity for most configurations, and depends on several plasma parameters. A scaling relation for the L$\mathrm{H}$ power threshold has been developed from an international database: $P_{\mathrm{LH}}(\mathrm{MW})=$ $0.042 n_{20}^{0.73} B_{\mathrm{T}}^{0.74} S^{0.98}$ [2], with $S$ being the plasma surface area. The L-H power threshold is further found to depend on the magnetic geometry: plasmas with the ion $\nabla \mathrm{B}$ drift directed towards the dominant separatrix X-point having a lower power threshold than plasmas with the ion $\nabla B$ drift directed away from the X-point [3,4]. Several mechanisms have been identified that may cause this dependence on magnetic geometry. Increased flow shear of the edge turbulence occurs naturally in plasmas with the ion $\nabla \mathrm{B}$ drift towards the X-point [5], and scrape-off-layer flows are observed to change in ways that may facilitate the $\mathrm{L}-\mathrm{H}$ transition in these plasmas [6,7]. 
New experimental measurements on the DIII-D tokamak show that, in addition to the above dependencies, the L-H transition power threshold varies strongly with the injected neutral beam torque and consequent toroidal plasma rotation. It is found that the $\mathrm{L}-\mathrm{H}$ power threshold increases significantly with co-current rotation for plasmas with the ion $\nabla \mathrm{B}$ drift towards or away from the $\mathrm{X}$ point. The mechanism for this dependence appears to lie in the edge turbulence dynamics.

\section{Dependence of the L-H Power Threshold on Injected Torque}

Systematic experiments were performed to examine the dependence of the L-mode to H-mode power threshold on rotation. These were facilitated by the reconfiguration of the neutral beam injection system on DIII-D [8] that now includes three co-current injection neutral beam lines (5 sources, $\left.\mathrm{P}_{\mathrm{co}} \leq 12.5 \mathrm{MW}\right)$ and one counter-current neutral beam line ( 2 sources, $\mathrm{P}_{\text {counter }} \leq 5 \mathrm{MW}$ ). Central toroidal rotation can be varied from $\sim 400 \mathrm{~km} / \mathrm{s}$ in the co-current direction to $\sim 150 \mathrm{~km} / \mathrm{s}$ in the counter current injection. It is noted that balanced neutral beam torque injection results in modest co-current toroidal rotation as a result of the unexplained but widely observed intrinsic rotation [9].

Plasmas for these experiments had $I_{\mathrm{p}}=1 \mathrm{MA}$, $B_{\mathrm{T}}=2 \mathrm{~T}, n_{\mathrm{e}, \mathrm{o}}=2-3 \times 10^{19} \mathrm{~m}^{-3}$ (in L-mode) and injected power ranging from $0-7 \mathrm{MW}$. The magnetic configuration was varied to examine plasmas with the ion $\nabla \mathrm{B}$ drift directed towards and away from the X-point; these configurations have been clearly demonstrated on DIII-D and other tokamaks to have a strong influence on the L-H power threshold. Equilibrium reconstructions for these two cases are shown in Fig. 1, along with an expanded view of several key diagnostics utilized.

The time histories of several parameters for two rotational conditions are presented in Fig. 2 for plasmas with the ion $\nabla \mathrm{B}$ drift directed away from the dominant X-point. In one plasma, the neutral beams were injected purely in the co-current direction, while in the other, the neutral beam torque was approximately balanced so the net injected torque was near zero. In both cases, the neutral beam power was increased gradually in approximately $400 \mathrm{~kW}$ steps to allow for a relatively precise determination of the L-H power threshold. Each step was maintained for $320 \mathrm{~ms}$, several times the energy confinement time and neutral beam ion slowing down time, allowing for the plasma to reach a new quasi-equilibrium state at each step. The co-injection plasma undergoes an L-H transition at $2.1 \mathrm{~s}$ (indicated by a dotted vertical bar) when the recycling light undergoes a rapid reduction. The power injected at this point was near $6 \mathrm{MW}$ [Fig. 2(b)] with a torque of near $4 \mathrm{~N}$-ms [Fig. 2(d)]. The core $(\mathrm{r} / \mathrm{a}=0.0)$

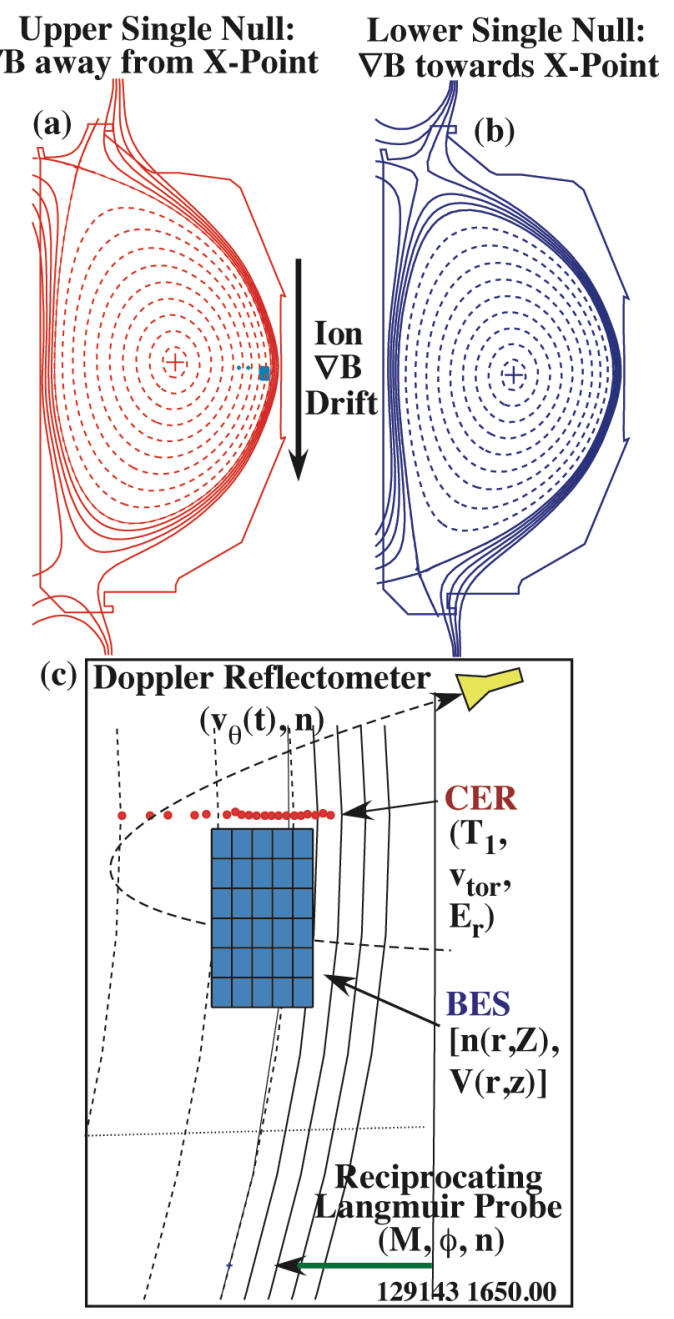

FIG. 1. Magnetic equilibria for plasmas with: (a) the ion NB drift away from (upper-single-null), and (b) towards (lowergle-null) the dominant separatrix $\mathrm{X}$ , (c) expanded view of primary charge exchange recombination spectroscopy (CER), beam emission spectroscopy (BES), and reciprocating Langmuir probe. 
and edge $(\mathrm{r} / \mathrm{a}=0.9)$ toroidal rotation velocities plotted in Fig. 2(c) show that the toroidal rotation increases significantly as the cocurrent injected beam power increases. For the near zero torque case, the toroidal rotation is more modest, but notably nonzero. The L-H power threshold differs by a factor of 2 with rotation, a dramatic difference not previously observed.

A large number of similar discharges were performed to examine the dependence of the L-H power threshold on injected neutral beam torque for different ion $\nabla \mathrm{B}$ drift directions. The results of these scans are shown in Fig. 3. The L-H power threshold is taken in each case as the average of the power level at which the transition occurred, and the previous (lower) power level step, since the power was gradually ramped up in these discharges.

The power flow required to induce a transition from L-mode to $\mathrm{H}$-mode plasmas is found to depend strongly on the injected neutral beam torque and consequent toroidal plasma rotation. The L-H power threshold in plasmas with the ion $\nabla \mathrm{B}$ drift away from the $\mathrm{X}$-point decreases from 4-6 MW with cocurrent beam injection, to 2-3 MW near zero net injected torque, and to $<2 \mathrm{MW}$ with counter injection, as shown in Fig. 3. Plasmas with the ion $\nabla \mathrm{B}$ drift towards the $\mathrm{X}$-point exhibit a qualitatively similar though less pronounced power threshold dependence on rotation.

In related experiments, H-modes were induced via application of electron cyclotron heating $(\mathrm{ECH})$ power to discharges with a $25 \%$ higher density than those shown in Fig. 3. The $\mathrm{H}$-mode power threshold is found to be similar for balanced neutral beam injection (NBI) and ECH-only discharges. ECH-only heated plasmas with the ion $\nabla \mathrm{B}$ drift directed towards the X-point actually have a modestly lower L-H power threshold than NBI-only heated discharges, while ECH-only heated plasmas with the ion $\nabla \mathrm{B}$ drift directed away from the $\mathrm{X}$ point have a higher L-H power threshold than NBI-only heated discharges. At these higher densities, a larger difference in the power threshold is observed between plasmas with the ion $\nabla \mathrm{B}$ drift towards and away from the X-point (ECH or NBI heating).

The difference in $\mathrm{L}-\mathrm{H}$ power threshold between plasmas with the ion $\nabla \mathrm{B}$ drift towards and away from the $\mathrm{X}$-point is dependent on the torque injection. For neutral beam injection in the co-current direction with ion $\nabla \mathrm{B}$ drift away from the $\mathrm{X}$-point, the $\mathrm{L}-\mathrm{H}$ power threshold is 
double the amount for the ion $\nabla \mathrm{B}$ drift towards the $\mathrm{X}$-point. This difference in power threshold persists at zero net injected torque but is substantially reduced to about a $25 \%$ difference.

\section{Turbulence Characteristics and Dynamics}

A leading hypothesis for the $\mathrm{L}-\mathrm{H}$ transition is that an increase in the shear in the radial electric field in the plasma edge decorrelates and tears apart turbulent eddies [10], leading to suppression of turbulence and turbulent transport in the edge, followed by increased edge pedestal pressure, and further increase in the radial electric field shear, reinforcing the edge H-mode pressure pedestal.

Here, we present measurements of density fluctuations associated with turbulence, poloidal flows and mode structure of that turbulence, and shear and decorrelation rates of the turbulence. 2D measurements of the turbulence in the $\mathrm{R}-\mathrm{Z}$ plane were obtained over the radial range $0.9<\mathrm{r} / \mathrm{a} \leq 1$ (extending slightly into the scrape-off-layer region) with the high-sensitivity beam emission spectroscopy (BES) diagnostic [11-13]. BES examines long-wavelength density fluctuations $\left(k_{\perp}<3 \mathrm{~cm}^{-1}\right)$ in the radialpoloidal plane. A $5 \times 6$ grid of BES channels is deployed near the outboard midplane, as shown in Fig. 1(c), covering a range of about $4.5 \mathrm{~cm}$ radially and $7.2 \mathrm{~cm}$ poloidally (each channel approximately $0.9 \mathrm{~cm}$ radially x $1.2 \mathrm{~cm}$ poloidally and arranged directly adjacent to each other). This arrangement provided measurements of the radial dependence, poloidal flows and flow shear, zonal flows and images and movies of the turbulence. Fluctuation measurements were also obtained with a newly implemented Doppler backscattering system [14] and a reciprocating Langmuir probe array.

Turbulence is rapidly suppressed at the L-H transition. Figure 4(a) shows a time and frequency resolved spectrogram of the density fluctuations near $\mathrm{r} / \mathrm{a}=0.95$ in a balanced-injection condition. The large reduction in turbulence amplitude at all frequencies is apparent at the L-H transition just before $1700 \mathrm{~ms}$. Turbulence is
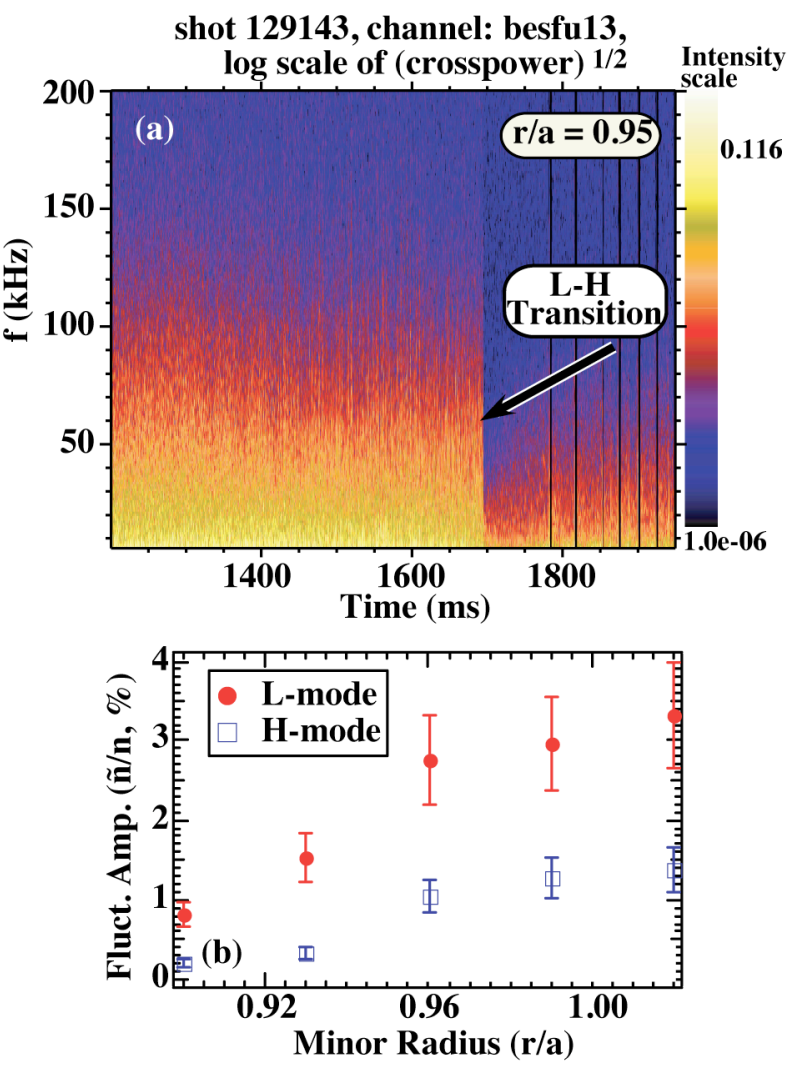

FIG. 4. Density fluctuation characteristics measured near the edge $(0.9<\mathrm{r} / \mathrm{a} \leq 1.0)$ of an uppersingle-null discharge: (a) frequency and time resolved spectrogram of density fluctuations at $\mathrm{r} / \mathrm{a}=0.95$ showing rapid fluctuation suppression at the L-H transition at $t=1696 \mathrm{~ms}$, (b) the integrated fluctuation amplitude edge profile in L- and $\mathrm{H}$-mode in a balanced-injection condition. suppressed within tens of microseconds after the transition, a few turbulence decorrelation times. The radial profile of fluctuation amplitudes before and after the transition in Fig. 4(b) shows the fluctuation amplitude reduction obtained by integrating the normalized fluctuation power over $10-250 \mathrm{kHz}$. 
The poloidal group velocity of the turbulence near the plasma edge show a strong dependence on toroidal rotation, as seen in Fig. 5. The advection of turbulent structures in the poloidal direction is measured by applying time-lag cross-correlation techniques to poloidally displaced BES channels [15]. Each spectrum is obtained in the 50 or $100 \mathrm{~ms}$ time window before the L-H transition. At $\mathrm{r} / \mathrm{a}=0.9$, the co-injection plasma exhibits significantly higher fluctuation power, and the fluctuations are Dopplershifted to near $400 \mathrm{kHz}$ largely by the local ExB velocity. The balanced injection plasma, which has significantly lower total injected power at this time frame, has lower fluctuation level and smaller frequency shift. The frequency-resolved phase shift between two poloidally displaced channels is shown in Fig. 5(c). The slope of this curve is inversely related to
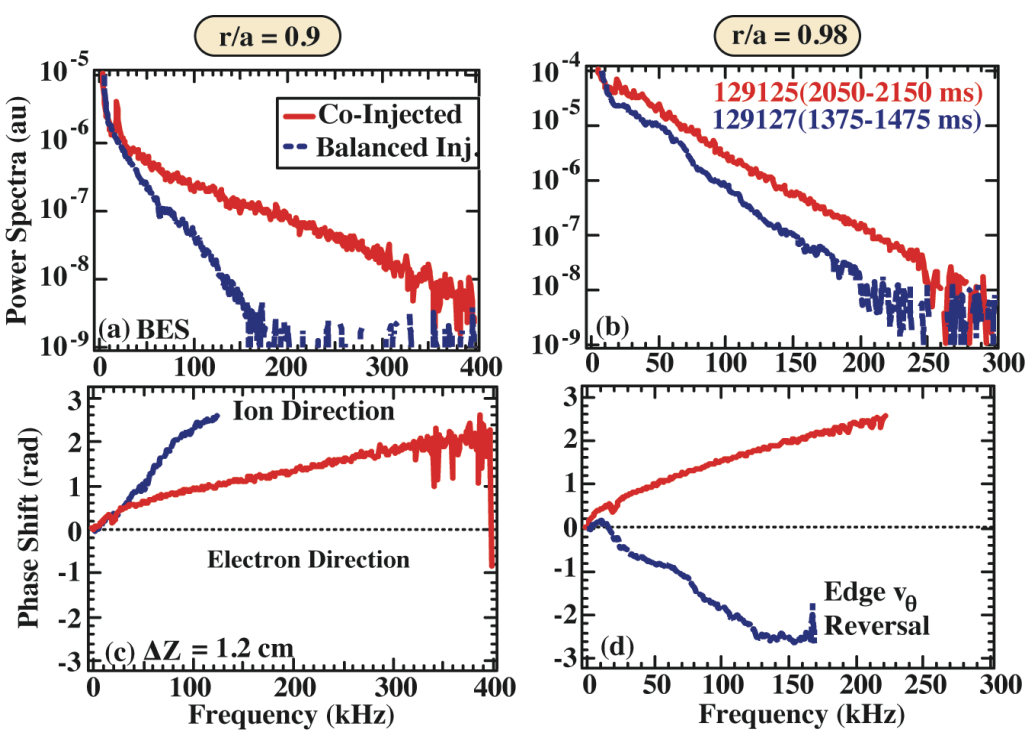

FIG. 5. Cross-power spectra and phase shift of poloidally displaced BES channels $(\Delta Z=1.2 \mathrm{~cm})$ at $\mathrm{r} / \mathrm{a}=0.9$ and $\mathrm{r} / \mathrm{a}=0.98$ in coinjection and balanced injection plasmas with the ion $\nabla \mathrm{B}$ drift directed away from the $\mathrm{X}$-point illustrating the flow reversal in the later case. the local poloidal group velocity of the advecting turbulent structures, so the co-injection plasma has more rapidly advecting eddies in the ion diamagnetic direction (co-current toroidal rotation on DIII-D results in a radial electric field with an ExB velocity in the ion diamagnetic direction). At $\mathrm{r} / \mathrm{a}=0.98$, the co-injection spectrum again has higher fluctuation power, with the spectrum Doppler-shifted to about $300 \mathrm{kHz}$, and the phase shift again exhibits advection in the ion diamagnetic direction at somewhat reduced poloidal velocity. The balanced-injection plasma exhibits a striking difference in that the turbulence is advecting in the electron diamagnetic direction, illustrated by the negative phase shift. This demonstrates that over $0.9<\mathrm{r} / \mathrm{a}<0.98$, the turbulence undergoes a poloidal flow reversal; this is not observed for the co-current plasma and suggests that a significant radial flow shear exists in the balanced-injection case.

\section{Zonal Flow Evolution Prior to Torque-Scan-Induced L-H Transition}

The robustness of the power threshold dependence on rotation was tested by attempting to induce an L-H transition by reducing the torque at constant power in a plasma with the ion $\nabla \mathrm{B}$ drift away from the X-point. $3 \mathrm{MW}$ of co-injected neutral beams were initially injected into the plasma, well below the L-H power threshold indicated in Fig. 3. The torque was varied from co-injection to balanced while maintaining constant power input, at which point an L-H transition occurred at $1.7 \mathrm{~s}$, when the injected power was nearly balanced at zero net torque (near when the transition could be expected based on the data in Fig. 3).

The fluctuation power spectra at $\mathrm{r} / \mathrm{a}=0.96$ are presented in Fig. 6 for three distinct times in this discharge: one in the co-injection phase, one with $3 / 4$ co-injected and 1/4 counterinjected power, and one at balanced injection, just prior to the L-H transition. The evolution of these basic characteristics is similar to that shown previously in Fig. 5 for separate coinjection and balanced-injection discharges except that here the injected power level is 

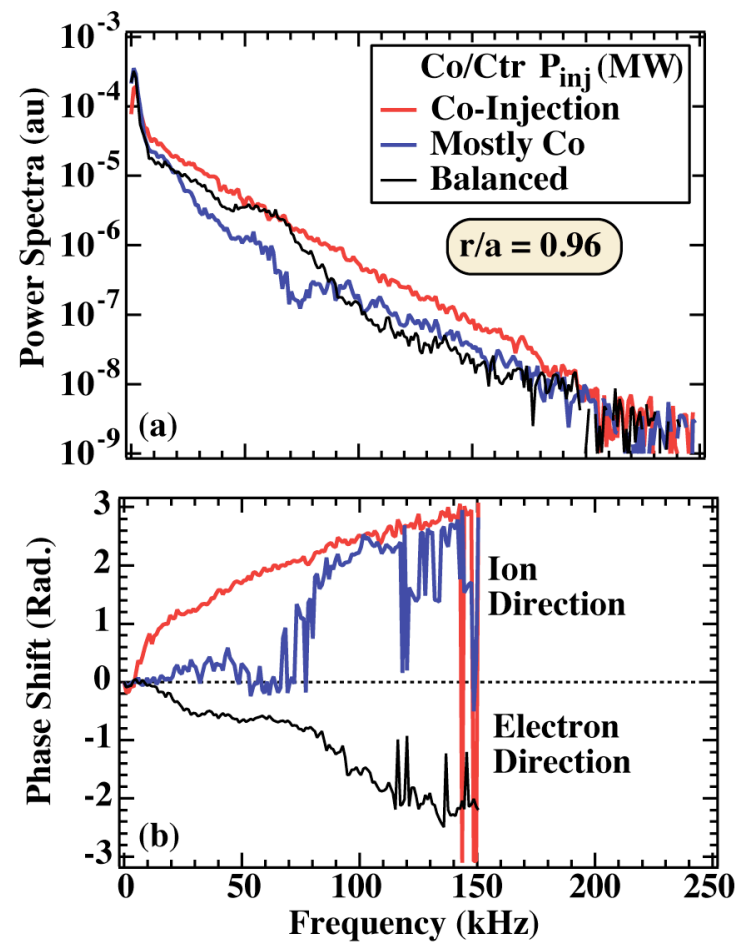

FIG. 6. (a) Cross power spectra and (b) phase shift of poloidally-displaced BES channels $(\Delta Z=1.2 \mathrm{~cm})$ at $\mathrm{r} / \mathrm{a}=0.96$ at three time intervals during the torque scan. The poloidal advection velocity of the turbulent eddies are observed to reverse flow from the ion diamagnetic direction with co-injection to the electron direction with balanced-injection. constant. The power spectra change as injected torque is reduced, with a transition from a unimodal structure in co-injection to a dual-mode structure as torque injection is reduced. The phase shift shows a clear reversal of the poloidal advection of fluctuations from the ion diamagnetic direction to the electron diamagnetic direction as the discharge evolves towards balanced injection. At the intermediate times, a flattening of the phase shift below $70 \mathrm{kHz}$ is a manifestation of the dual-mode structure.

The behavior of zonal flows [16] was examined in this torque scan discharge using the 2D BES measurements. Zonal flows, toroidally and poloidally symmetric $(n=0, m=0)$, radially localized electrostatic potential fluctuations, are thought to play a role in triggering the L-H transition [17]. Poloidally displaced BES measurements are used here to examine the highfrequency poloidal velocity spectrum by applying time-delay-estimation techniques [18-20].

The measured velocity fluctuation spectrum is shown for various times in Fig. 7 at a location of $\mathrm{r} / \mathrm{a}=0.9$ during the torque scan discharge. Figure 7(a) shows the spectrum for three time intervals during the co-injection phase and the beginning of the counterinjection phase. The coherent oscillation is the GAM, with a well-defined frequency near the predicted frequency of $f_{\mathrm{GAM}}=c_{\mathrm{s}} / 2 \pi R$. While initially clear, the GAM decays rapidly in amplitude as the plasma rotation is reduced. Later in time, after the GAM has effectively disappeared, the velocity fluctuation spectrum exhibits a broad, low-frequency structure for $f<10 \mathrm{kHz}$ seen in Fig. 7(b). This structure appears strikingly similar to the Zero-Mean-Frequency-Zonal Flow (ZMF-ZF) that has been previously observed [21] deeper in the plasma core $(\mathrm{r} / \mathrm{a}<0.8)$. The larger relative power and the lower-frequency of the ZMF-ZF structure are more likely to have an impact on the edge turbulence than the higher frequency GAM [22]. It is speculated that this ZMF-ZF structure might help trigger the L-H transition and may also play a role in the observed ion and electron directed advection of the turbulent eddy structure.

\section{Radial Electric Field}

The gradient in the electric field is especially crucial to the $\mathrm{L}-\mathrm{H}$ transition as the radially sheared ExB velocity decorrelates and suppresses turbulence, leading to the transition. $E_{\mathrm{r}}(r)$ is determined from the edge plasma pressure gradient and velocity through the force balance equation: $E_{\mathrm{r}}=\nabla P / Z_{\mathrm{I}} e n_{\mathrm{I}}+v_{\phi, \mathrm{i}} B_{\theta}-v_{\theta, \mathrm{i}} B_{\phi}[10]$. All quantities in this equation are measured for the carbon impurity species with an array of high spatial resolution and high-density charge exchange recombination spectroscopy channels [Fig. 1(c)]. Here, the interplay of the pressure gradient and rotation terms in the ion force balance equation conspire to facilitate increased $E_{r}$ shear at lower rotation, while reducing $E_{r}$ shear at similar injected power in highly corotating discharges. 
The radial electric field structure, $E_{\mathrm{r}}(r)$, near the plasma edge has been examined for several discharge conditions, as shown in Fig. 8 for plasmas with the ion $\nabla \mathrm{B}$ drift away from the X-point at times shortly prior to the L-H transition. For the balanced-injection plasma, shown in Fig. 8(a), the edge $E_{\mathrm{r}}(r)$ and $E_{\mathrm{r}}^{\prime}(r)$ is dominated by the pressure gradient term, and a significant $E_{\mathrm{r}}(r)$ gradient is observed near the plasma edge. The gradient near the separatrix is thought to help precipitate the L-H transition. In the case of the co-injection plasma, shown in Fig. 8(b), $E_{\mathrm{r}}(r)$ is dominated by the toroidal velocity term, and there is no clear gradient very close to the separatrix. Rather, a large gradient is built up near $\psi=0.96$ (note that $\psi$, the normalized poloidal flux, is used as the radial coordinate here rather than the normalized toroidal flux, r/a, used elsewhere), somewhat inboard of the steep gradient region in the balanced injection case. For the co-injection plasma at a lower injected power level of 4.1 $\mathrm{MW}$, the $E_{\mathrm{r}}(r)$ profile is featureless in the near edge region with no sharp gradient region [Fig. 8(c)]. These results are similar to the turbulence and turbulence poloidal velocity profile measurements [23] and demonstrate a consistent picture for how the plasma edge develops and evolves significantly differently in the two rotation conditions. Furthermore, these key differences suggest a causal relationship for the lower L-H power threshold results in the balanced injection case relative to co-injection.
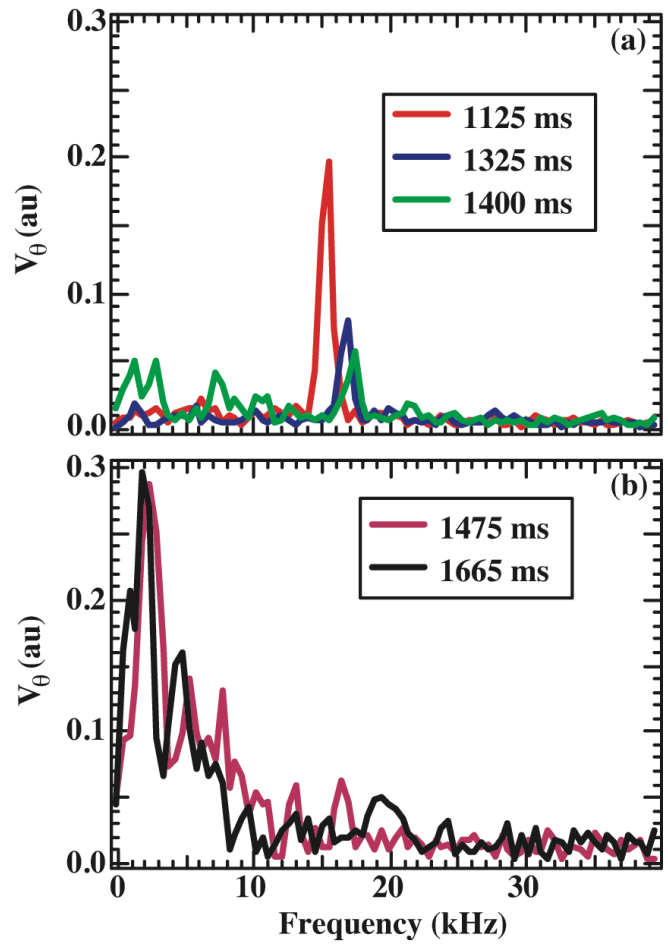

FIG. 7. Turbulence poloidal velocity spectrum showing evolution of zonal flow structures: (a) three times in the coinjection and 3/4-co, 1/4-counter injection phases showing GAM zonal flow oscillation, and (b) two later times as the plasma approached balanced injection, exhibiting the disappearance of the GAM and appearance of a structure with appearance of a zero-mean-frequency zonal flow.
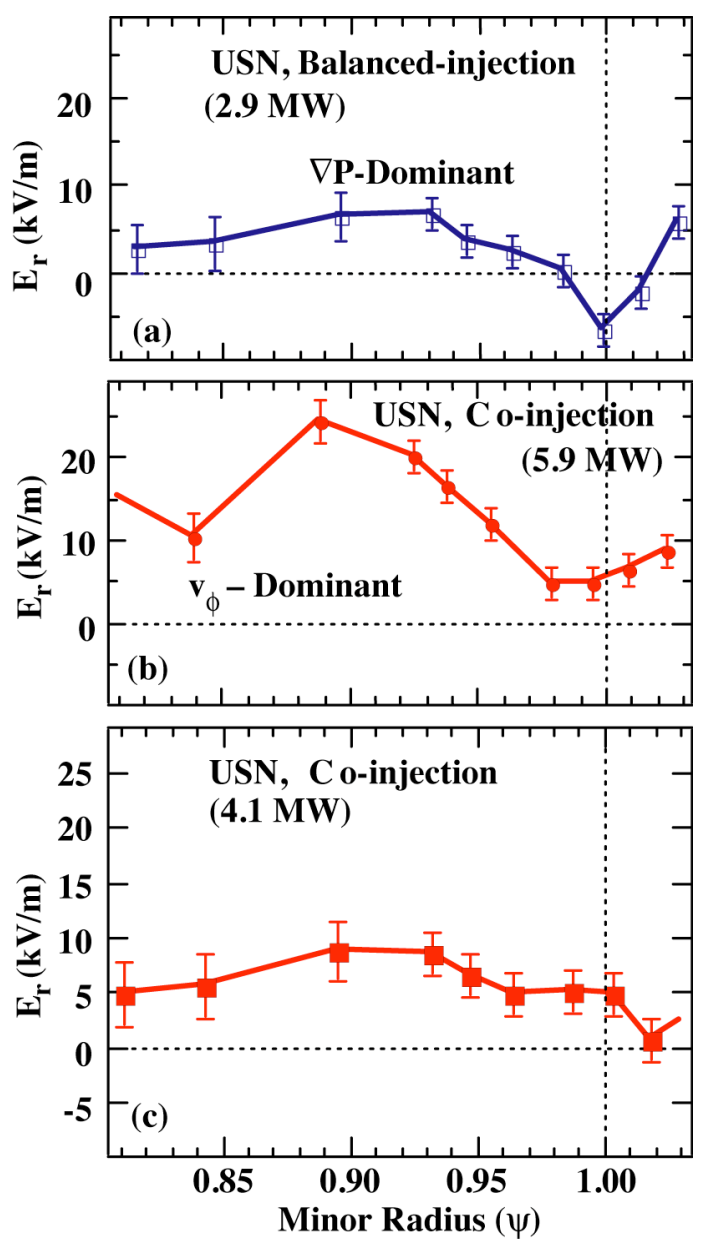

FIG. 8. Edge radial electric field just prior to the $\mathrm{L}-\mathrm{H}$ transition in plasmas with ion $\nabla \mathrm{B}$ drift directed away from the X-point (USN) in a: (a) balanced-injection plasma, showing a sharp pressure-gradient driven $E_{\mathrm{r}}$ well near the plasma edge, and (b) co-injection plasma with little edge gradient and a sharper gradient inboard; (c) co-injected plasma at lower power level show little gradient. 


\section{Summary and Conclusions}

Scaling relations for the L-H power threshold do not presently include a rotational dependence, yet these results demonstrate that toroidal rotation is a critical parameter affecting the threshold. The mechanism for this effect appears to lie in the interplay of the radial electric field, turbulence flows, fluctuation spectral structure and the zonal flows. This reduced power threshold at lower toroidal rotation may benefit large low-rotation plasmas such as ITER.

The L-mode to H-mode power threshold in DIII-D has been shown to depend sensitively on the injected neutral beam torque and consequent toroidal rotation of the plasma. This dependence is found to apply to plasmas with the ion $\nabla \mathrm{B}$ drift directed towards and away from the dominant $\mathrm{X}$-point, with a stronger dependence found for plasmas with the ion $\nabla \mathrm{B}$ drift directed away from the X-point.

The mechanism for this significant difference in $P_{\mathrm{LH}}$ with rotation appears to reside in a complex interplay of the edge turbulence, turbulence flows and flow shear, mode structure, zonal flow dynamics and radial electric field. Two-dimensional turbulence measurements are obtained near the plasma edge with the Beam Emission Spectroscopy, which show dramatic and significant changes in the edge fluctuation spectra and flows with changing toroidal rotation. These results have potentially beneficial implications for slowly rotating plasmas, such as those that will be encountered on the ITER device.

\section{Acknowledgment}

This work was supported by the US Department of Energy under DE-FG02-89ER53296, DEFC02-04ER54698, DE-FG02-07ER54917, DE-FG03-01ER54615，DE-AC02-76CH03073, and DE-AC52-07NA27344.

\section{References}

[1] WAGNER, F., et al., Phys. Rev. Lett. 49, 1408 (1982).

[2] DOYLE, E. J., et al., Nucl. Fusion 47, S18 (2007).

[3] CARLSTROM, T.N., Plasma Phys. Control. Fusion 44, A333 (2002).

[4] CARLSTROM, T.N., Fusion Sci. Tech. 48, 997 (2005).

[5] FENZI, C., Phys. Plasmas 12, 062307 (2005).

[6] LABOMBARD, B., et al., Phys. Plasmas 12, 056111 (2005).

[7] AYDEMIR, A.Y., Phys. Rev. Lett. 98, 225002 (2007).

[8] LUCE, T.C., et al., in Proc. 21st IAEA Fusion Energy Conf., Chengdu, China (2006).

[9] RICE, J.E., et al., Nucl. Fusion 44, 379 (2004).

[10] BURRELL, K.H., Plasmas 4, 1499 (1997).

[11] GUPTA, D., et al., Rev. Sci. Instrum. 75, 3493 (2004).

[12] FONCK, R.J., et al., Rev. Sci. Instrum. 61, 3487 (1990).

[13] McKEE, G.R., et al., Plasma Fusion Res. 2, S1025 (2007).

[14] SCHMITZ, L., et al., Rev. Sci. Instrum. 79, ?? (2008)

[15] DURST, R., et al., Rev. Sci. Instrum. 63, 4907 (1992).

[16] DIAMOND, P.H., et al., Plasma Phys. Control. Fusion 47, R35 (2005).

[17] KIM, E.J. and DIAMOND, P.H., Phys. Rev. Lett. 90, 185006 (2003).

[18] FUJISAWA, A., et al., Phys. Rev. Lett. 93, 165002 (2004).

[19] McKEE, G.R., et al., Plasma Phys. Control. Fusion 48, S123 (2006).

[20] JAKUBOWSKI, M., et al., Rev. Sci. Instrum. 72, 996 (2001).

[21] GUPTA, D.K., et al., Phys. Rev. Lett. 97, 125002 (2006).

[22] HAHM, T.S., et al., Phys. Plasmas 6, 922 (1999).

[23] SCHLOSSBERG, D.J., et al., "Dependence of the Low to High Confinement Mode Transition Power Threshold on Injected Torque," submitted Phys. Rev. Lett. (2007). 